\title{
Optimal matter-wave gravimetry
}

\author{
Michail Kritsotakis, ${ }^{1, *}$ Stuart S. Szigeti ${ }^{2,3}$ Jacob A. Dunningham, ${ }^{1}$ and Simon A. Haine ${ }^{1,2}$ \\ ${ }^{1}$ Department of Physics and Astronomy, University of Sussex, Brighton BN1 9QH, United Kingdom \\ ${ }^{2}$ Department of Quantum Science, Research School of Physics and Engineering, The Australian National University, \\ Canberra ACT 2601, Australia \\ ${ }^{3}$ Department of Physics, Centre for Quantum Science, and Dodd-Walls Centre for Photonic and Quantum Technologies, \\ University of Otago, Dunedin 9010, New Zealand
}

(Received 17 October 2017; published 27 August 2018)

\begin{abstract}
We calculate quantum and classical Fisher information for gravity sensors based on matter-wave interference, and find that current Mach-Zehnder interferometry is not optimally extracting the full metrological potential of these sensors. We show that by making measurements that resolve either the momentum or the position we can considerably improve the sensitivity. We also provide a simple modification that is capable of more than doubling the sensitivity.
\end{abstract}

DOI: 10.1103/PhysRevA.98.023629

Atom interferometry is a leading inertial-sensing technology, having demonstrated state-of-the-art gravimetry [1-7] and gradiometry [8-14] measurements. Nevertheless, orders of magnitude improvement in sensitivity is required for applications in navigation [15] and mineral exploration [16], as well as improved tests of the equivalence principle [17-19] and quantum gravity [20,21]. For the commonly used Mach-Zehnder [i.e., Kasevich-Chu (KC)] configuration [22,23], semiclassical calculations [24-27] reveal that the matter wave accrues relative phase $\phi=\mathbf{g} \cdot \mathbf{k}_{L} T_{\pi}^{2}$, where $\mathbf{g}$ is the gravitational acceleration, $\hbar \mathbf{k}_{L}$ is the momentum separation of the two arms, and $2 T_{\pi}$ is the total interrogation time. Assuming $N$ uncorrelated particles, a population-difference measurement at the interferometer output yields sensitivity,

$$
\Delta g=\frac{1}{\sqrt{N} k_{0} T_{\pi}^{2}},
$$

where $k_{0}$ is the component of $\mathbf{k}_{L}$ aligned with $\mathbf{g}$. Equation (1) implies only four routes to improved sensitivity: (1) increase interrogation time, (2) increase the momentum separation of the arms (e.g., via large momentum transfer beam splitters [28-32]), (3) increase the atom flux, and/or (4) surpass the shot-noise limit with quantum correlations [33-37]. Although all routes are worth pursuing, each has unique limitations. For instance, size, weight, and power constraints limit both $T_{\pi}$ and the maximum momentum transferrable via laser pulses. Additionally, evaporative-cooling losses and momentum width requirements constrain atom fluxes [38-42]. Increases with number-conserving feedback cooling are possible, but untested [43-45]. Finally, quantum-correlated states must be compatible with the requirements of high-precision metrology [5,46-57] (e.g., high atom flux, low phase diffusion), and will only be advantageous if classical noise sources (e.g., $[58,59]$ ) are sufficiently controlled to yield shot-noise-limited operation prior to quantum enhancement.

\footnotetext{
*M.Kritsotakis@ sussex.ac.uk
}

This assessment assumes that Eq. (1) is the optimal sensitivity. In this article, we prove this conventional wisdom false by showing that matter-wave interferometers can attain better sensitivities than Eq. (1). Ultimately, the gravitational field affects the quantum state beyond the creation of a simple phase shift. We show this additional metrological potential via the quantum Fisher information (QFI), which determines the best possible sensitivity. We further determine the set of measurements required to attain this optimal sensitivity via the classical Fisher information (CFI). Our analysis reveals additional routes to improved sensitivity, such as variations in the measurement procedure and input source, and these should be considered when designing future matter-wave gravimeters. We also present a modified interferometer that more than doubles the sensitivity for the same interrogation time and momentum separation.

The focus of this article is KC interferometry based on state-changing Raman transitions, although our results also hold for Bragg transitions [5] and Bloch oscillations [29] in the appropriate regime. A KC interferometer is schematically depicted in Fig. 1(a). At time $t=0$ atoms with two internal states $|a\rangle$ and $|b\rangle$, initially in $|a\rangle$, are excited to an equal superposition of $|a\rangle$ and $|b\rangle$ via a coherent $\pi / 2$ pulse. Atoms transferred to $|b\rangle$ also receive a momentum kick $\hbar k_{0}$. At $t=T_{\pi}$, a $\pi$ pulse acts as a mirror, before the two matter waves are interfered at $t=T=2 T_{\pi}$ by a second $\pi / 2$ pulse.

\section{QFI FOR A PARTICLE IN A GRAVITATIONAL FIELD}

The quantum Cramér-Rao bound (QCRB) gives a lower bound on the sensitivity [60]. For $N$ uncorrelated particles this is $\Delta g^{2} \geqslant 1 /\left(N F_{Q}\right)$, where $F_{Q}$ is the single-particle QFI [61-63], which for a pure single-particle state $|\Psi\rangle$ is

$$
F_{Q}=4\left(\left\langle\partial_{g} \Psi \mid \partial_{g} \Psi\right\rangle-\left|\left\langle\Psi \mid \partial_{g} \Psi\right\rangle\right|^{2}\right) \text {. }
$$

For the KC interferometer, semiclassical arguments give $|\Psi\rangle=\frac{1}{\sqrt{2}}\left(|a\rangle+e^{i g k_{0} T^{2}}|b\rangle\right)$ before the final beam splitter and a QFI $F_{Q}^{\mathrm{sc}}=k_{0}^{2} T_{\pi}^{4}$ [64], consistent with Eq. (1). However, 

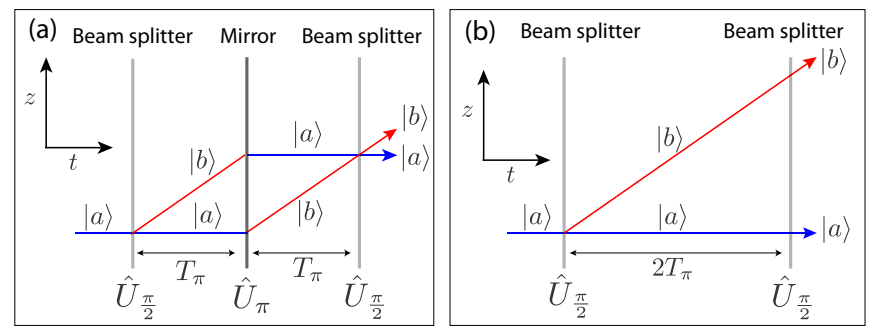

FIG. 1. Space-time diagrams for (a) KC interferometry and (b) Ramsey interferometry (no mirror pulse), which are both sensitive to gravitational fields and accelerations.

this derivation treats the particle's motion semiclassically, neglecting the noncommutability of position and momentum. We account for this here. For the moment we consider only the center-of-mass degrees of freedom. In the presence of a uniform gravitational field $g$ acting along the $z$ axis, a particle of mass $m$ in state $\left|\psi_{0}\right\rangle$ evolves to $|\psi(T)\rangle=\hat{U}_{g}\left|\psi_{0}\right\rangle$ after time $T$, where $\hat{U}_{g}=\exp \left[-\frac{i T}{\hbar}\left(\frac{\hat{\mathbf{p}}^{2}}{2 m}+m g \hat{z}\right)\right]$. As shown in Appendix A, we can rewrite

$$
\hat{U}_{g}=e^{-i \frac{T}{\hbar} \frac{\hat{p}^{2}}{2 m}} e^{-i g \hat{G}_{0}(T)} e^{i \frac{m g^{2} T^{3}}{12 \hbar}},
$$

where

$$
\hat{G}_{0}(T)=\frac{T}{\hbar}\left(\frac{T}{2} \hat{p}_{z}+m \hat{z}\right) .
$$

The QFI is

$$
\begin{aligned}
F_{Q}(T) & =4 \operatorname{Var}\left(G_{0}(T)\right) \\
& =\frac{T^{4}}{\hbar^{2}} \operatorname{Var}\left(p_{z}\right)+\frac{4 m^{2} T^{2}}{\hbar^{2}} \operatorname{Var}(z)+\frac{4 m T^{3}}{\hbar^{2}} \operatorname{Cov}\left(p_{z}, z\right),
\end{aligned}
$$

where the variances and covariance are evaluated with respect to $\left|\psi_{0}\right\rangle$. To compare Eq. (5a) and $F_{Q}^{\text {sc }}$, consider a state $\left|\psi_{0}\right\rangle$ with two well-defined peaks in momentum space separated by $\hbar k_{0}$, giving $\operatorname{Var}\left(p_{z}\right) \approx\left(\hbar k_{0}\right)^{2}$. For sufficiently large $k_{0}$ and $T$ such that $\left(\hbar k_{0} T / 2\right)^{2} \gg m^{2} \operatorname{Var}(z), m T \operatorname{Cov}\left(p_{z}, z\right)$, the first term of Eq. (5b) dominates, and $F_{Q}\left(2 T_{\pi}\right) \approx k_{0}^{2} T_{\pi}^{4}=F_{Q}^{\mathrm{sc}}$. However, the additional terms in Eq. (5b) potentially allow sensitivities better than Eq. (1).

\section{QFI FOR KC INTERFEROMETRY}

Equation (5a) is not the QFI for a KC interferometer, as we must account for the internal state degrees of freedom as well as the action of the mirror pulse. The evolution is given by

$$
\hat{U}_{\mathrm{KC}}=\hat{U}_{\frac{\pi}{2}}^{\phi_{3}} \hat{U}_{g}\left(T_{2}\right) \hat{U}_{\pi}^{\phi_{2}} \hat{U}_{g}\left(T_{1}\right) \hat{U}_{\frac{\pi}{2}}^{\phi_{1}},
$$

where

$$
\hat{U}_{\theta}^{\phi}=\hat{1} \cos \left(\frac{\theta}{2}\right)-i\left(|b\rangle\langle a| e^{i\left(k_{0} \hat{z}-\phi\right)}+\text { H.c. }\right) \sin \left(\frac{\theta}{2}\right)
$$

governs the beam splitter and mirror dynamics. As shown in Appendix D, Eq. (7) is an excellent approximation to the beam splitting and mirror dynamics when the pulse duration is much shorter than the time scale for atomic motional dynamics. Here
$T_{1(2)}$ are evolution times before(after) the $\pi$ pulse and $\phi$ is the pulse phase, controlled via the relative phase of the two Raman lasers. The first $\pi / 2$ pulse maps the initial state $\left|\Psi_{0}\right\rangle=|a\rangle\left|\psi_{0}\right\rangle$ to $\left|\Psi_{0}^{\prime}\right\rangle=\hat{U}_{\frac{\pi}{2}}^{\phi_{1}}\left|\Psi_{0}\right\rangle=\frac{1}{\sqrt{2}}\left(|a\rangle-i e^{i\left(k_{0} \hat{z}-\phi_{1}\right)}|b\rangle\right)\left|\psi_{0}\right\rangle$, where $\left|\psi_{0}\right\rangle$ contains the initial state's motional degrees of freedom. As detailed in Appendix B,

$$
|\Psi(T)\rangle=\hat{U}_{\mathrm{KC}}\left|\Psi_{0}\right\rangle=\hat{U}_{0} e^{-i g\left(\hat{G}_{0}(T)+\hat{G}_{e}\right)}\left|\Psi_{0}^{\prime}\right\rangle,
$$

where

$$
\begin{aligned}
& \hat{G}_{e}=\hat{S}_{z} k_{0} T_{2}^{2}, \\
& \hat{S}_{z}=\frac{1}{2}(|a\rangle\langle a|-| b\rangle\langle b|), \\
& \hat{U}_{0}=\hat{U}_{\frac{\pi}{2}}^{\phi_{3}} e^{-i \frac{T_{2}}{\hbar} \frac{\hat{p}^{2}}{2 m}} \hat{U}_{\pi}^{\phi_{2}} e^{-i \frac{T_{1}}{\hbar} \frac{\hat{p}^{2}}{2 m}},
\end{aligned}
$$

and $T=T_{1}+T_{2}$, giving QFI,

$$
F_{Q}^{\mathrm{KC}}(T)=4 \operatorname{Var}\left(G_{0}(T)\right)+\frac{1}{4} k_{0}^{2}\left(T^{2}-2 T_{2}^{2}\right)^{2},
$$

where $\operatorname{Var}\left(G_{0}(T)\right)$ is taken with respect to $\left|\psi_{0}\right\rangle$. For $T_{1}=T_{2}=$ $T_{\pi}$,

$$
F_{Q}^{\mathrm{KC}}(T)=4 \operatorname{Var}\left(G_{0}(T)\right)+k_{0}^{2} T_{\pi}^{4} .
$$

Since $\operatorname{Var}\left(G_{0}(T)\right) \geqslant 0$, this implies $F_{Q}^{\mathrm{KC}} \geqslant F_{Q}^{\mathrm{sc}}$, thereby permitting sensitivities better than Eq. (1).

\section{CLASSICAL FISHER INFORMATION}

Although the QFI gives the best possible sensitivity, it is silent on how to achieve this sensitivity. The attainable sensitivity for a particular measurement choice is given by the CFI, which quantifies the information contained in the probability distribution constructed from measurements of a particular observable, and necessarily depends upon this choice of observable. We calculate the CFI via

$$
F_{C}(\hat{\Lambda})=\int d \lambda \frac{\left[\partial_{g} P(\lambda)\right]^{2}}{P(\lambda)},
$$

where $P(\lambda)$ is the probability of obtaining result $\lambda$ when the observable $\hat{\Lambda}$ is measured [61,62]. The CFI is bounded by the QCRB $F_{C} \leqslant F_{Q}$, so a measurement that saturates this bound is the optimal measurement.

\section{A. CFI for population-difference measurement}

For the standard population-difference measurement at the $\mathrm{KC}$ interferometer output, $\hat{\Lambda}=\hat{S}_{z}$ and $F_{C}\left(\hat{S}_{z}\right)=$ $\sum_{s=a, b}\left(\partial_{g} P_{s}\right)^{2} / P_{s}$, where $P_{s}=\int d z \mid\left\langle s|\langle z \mid \Psi(T)\rangle|^{2}\right.$. As detailed in Appendix $\mathrm{C}$, an analytic solution exists in this case. Specifically,

$$
\begin{aligned}
& P_{a}=\frac{1}{2}(1+|\mathcal{C}| \sin \alpha), \\
& P_{b}=\frac{1}{2}(1-|\mathcal{C}| \sin \alpha),
\end{aligned}
$$

yielding

$$
F_{C}\left(\hat{S}_{z}\right)=\frac{|\mathcal{C}|^{2} \cos ^{2} \alpha}{1-|\mathcal{C}|^{2} \sin ^{2} \alpha} k_{0}^{2}\left(\frac{T^{2}}{2}-T_{1}^{2}\right)^{2},
$$




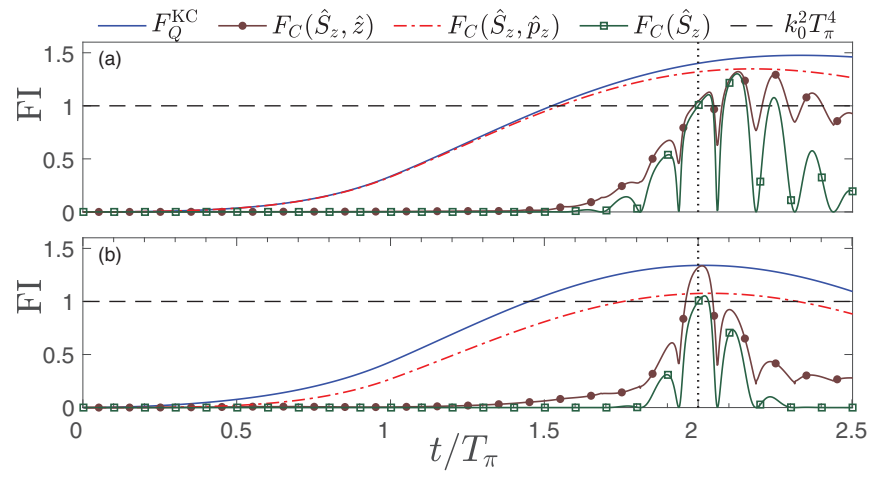

FIG. 2. Fisher information (FI) for $|\Psi(t)\rangle=\hat{U}_{\mathrm{KC}}(t)\left|\Psi_{0}\right\rangle$, where $T_{1}=t$ and $T_{2}=0$ for $t \leqslant T_{\pi}$, otherwise $T_{1}=T_{\pi}$ and $T_{2}=$ $t-T_{\pi}$, with initial Gaussian motional state (a) $\left\langle z \mid \psi_{0}\right\rangle=$ $\exp \left(-z^{2} / 2 \sigma^{2}\right) /\left(\pi \sigma^{2}\right)^{1 / 4}$ and (b) $\left\langle z \mid \psi_{0}\right\rangle=e^{-\left(\frac{1}{4}+i\right) z^{2} / 2 \sigma^{2}} /\left[\pi(2 \sigma)^{2}\right]^{1 / 4}$. FI has units $k_{0}^{2} T_{\pi}^{4}$, so when FI $>1$ a given measurement scheme achieves a sensitivity better than that predicted by the semiclassical limit Eq. (1). The QFI $F_{Q}^{\mathrm{KC}}$ gives the maximum possible FI. Here $\sigma=10 L$ and $T_{\pi}=100 t_{0}$, while the length $\left(L=k_{0}^{-1}\right)$ and time $\left(t_{0}=m / \hbar k_{0}^{2}\right)$ units depend on $k_{0}$.

where

$$
\begin{aligned}
\mathcal{C} & =\left\langle\psi_{0}\left|e^{i \frac{k_{0}}{m}\left(T_{2}-T_{1}\right) \hat{p}_{z}}\right| \psi_{0}\right\rangle \equiv|\mathcal{C}| e^{i \vartheta}, \\
\alpha & =\phi_{f}-\phi_{g}+\vartheta
\end{aligned}
$$

with $\phi_{f}=\frac{\hbar k_{0}^{2}}{2 m}\left(T_{2}-T_{1}\right)$ and $\phi_{g}=k_{0} g\left(\frac{T^{2}}{2}-T_{1}^{2}\right)$. The contrast $|\mathcal{C}|$ is determined by the spatial overlap of the two output wave packets, since $\frac{\hbar k_{0}}{m}\left(T_{2}-T_{1}\right)$ is the spatial separation. This depends strongly on the time difference $T_{2}-T_{1}$. For an initial Gaussian state $\left\langle z \mid \psi_{0}\right\rangle=\exp \left(-z^{2} / 2 \sigma^{2}\right) /\left(\pi \sigma^{2}\right)^{1 / 4}$, $|\mathcal{C}|=\exp \left[-\frac{\hbar^{2} k_{0}^{2}}{4 m^{2} \sigma^{2}}\left(T_{2}-T_{1}\right)^{2}\right]$.

Figure 2(a) shows the time dependence of the QFI and $F_{C}\left(\hat{S}_{z}\right)$ for this initial Gaussian state. Here $t=T_{1}+T_{2}$, we fix $T_{\pi}$ so the mirror pulse always occurs at $t=T_{\pi}$, and the second beam splitter occurs instantaneously before measurement. Explicitly, if $t \leqslant T_{\pi}$, then $T_{1}=t, T_{2}=0$, and the mirror pulse has no meaningful effect; if $t>T_{\pi}$ then $T_{1}=T_{\pi}$ and $T_{2}=$ $t-T_{\pi}$. When $T_{1}$ and $T_{2}$ are significantly different, the spatial overlap of the two modes at the interferometer output is poor, so both the contrast and CFI are close to zero. However, $|\mathcal{C}|=1$ when $T_{1}=T_{2}$ and $F_{C}\left(\hat{S}_{z}\right)=F_{Q}^{\mathrm{sc}}=k_{0}^{2} T_{\pi}^{4}$, giving the same sensitivity as Eq. (1). This is still less than $F_{Q}^{\mathrm{KC}}$, indicating that a different measurement could yield improved sensitivities.

\section{B. CFI for momentum-distribution measurement}

Now consider a measurement that distinguishes internal states and fully resolves the $z$ component of the final momentum distribution, such as reported in Ref. [65]. This measurement yields CFI,

$$
F_{C}\left(\hat{S}_{z}, \hat{p}_{z}\right)=\sum_{s=a, b} \int d p_{z} \frac{\left[\partial_{g} P_{s}\left(p_{z}\right)\right]^{2}}{P_{s}\left(p_{z}\right)},
$$

where $P_{s}\left(p_{z}\right)=\mid\left\langle s\left|\left\langle p_{z} \mid \Psi(T)\right\rangle\right|^{2}\right.$. Although no analytic formula exists for $F_{C}\left(\hat{S}_{z}, \hat{p}_{z}\right)$, the probabilities can be determined by numerically solving the Schrödinger equation, and the CFI computed from finite differences of these probabilities [63]. This requires an explicit choice of $g$; although we consider the sensitivity near $g=0$ for all numerical calculations, a large offset in $g$ is easily accounted for by adjusting the beam splitter phases, as in typical atomic gravimeters [41].

Figure 2(a) shows that $F_{C}\left(\hat{S}_{z}, \hat{p}_{z}\right)$ is significantly larger than $F_{C}\left(\hat{S}_{z}\right)$ and very close to $F_{Q}^{\mathrm{KC}}$. Additionally, $F_{C}\left(\hat{S}_{z}, \hat{p}_{z}\right) \approx$ $F_{Q}^{\mathrm{KC}}$ even when $T_{1}$ and $T_{2}$ are vastly different. This is because $P_{S}\left(p_{z}\right)$ displays interference fringes that are not present in $P_{s}=\int d p_{z} P_{s}\left(p_{z}\right)$ when spatial overlap is poor.

The origin of the increased information in $F_{C}\left(\hat{S}_{z}, \hat{p}_{z}\right)$ compared with $F_{C}\left(\hat{S}_{z}\right)$ is easily understood. Additional to the CFI associated with population exchange (generated by $\hat{G}_{e}$ ), there is information due to a shift in the momentum distribution. Concretely, consider initial momentum distribution $P_{0}\left(p_{z}\right)$. Under gravity, $\hat{p}_{z}(t)=\hat{p}_{z}(0)+m g t$, so $P\left(p_{z}, t\right)=P_{0}\left(p_{z}-\right.$ $m g t$ ), giving

$$
\begin{aligned}
F_{C}\left(p_{z}\right) & =\int d p_{z} \frac{\left[\partial_{g} P\left(p_{z}, t\right)\right]^{2}}{P\left(p_{z}, t\right)} \\
& =\left[\partial_{g} p_{z}(t)\right]^{2} \int d p_{z} \frac{\left[\partial_{p_{z}} P_{0}\left(p_{z}\right)\right]^{2}}{P_{0}\left(p_{z}\right)} \\
& \equiv(m t)^{2} F_{C}^{p_{z}}
\end{aligned}
$$

where $F_{C}^{p_{z}}$ is the CFI associated with resolvable small shifts in the momentum distribution. For the initial Gaussian considered in Fig. 2(a), adding this additional CFI to $F_{C}\left(\hat{S}_{z}\right)$ gives $\left.F_{C}\left(\hat{S}_{z}, \hat{p}_{z}\right)\right|_{2 T_{\pi}}=F_{Q}^{\mathrm{sc}}+8\left(m T_{\pi} \sigma / \hbar\right)^{2}$, in perfect agreement with our numerics. Note that this additional information is not the result of a phase shift so, unlike a standard $\mathrm{KC}$ interferometer, it is not affected by additional phase noise.

Our simulations also find near-perfect correlations between internal and momentum states, so a measurement that only resolves momentum (and not $\hat{S}_{z}$ ) also has CFI approximating $\left.F_{C}\left(\hat{S}_{z}, \hat{p}_{z}\right)\right|_{2 T_{\pi}}$, since an atom's internal state is inferred from its final momentum. Our analysis therefore holds for interferometers that do not change internal states, such as Bragg-scattering-based interferometers, provided $\hbar k_{0} \gg \delta p$, where $\delta p$ is the wave packet's initial momentum width [5,28]. In our simulations $\hbar k_{0} \approx 14 \delta p$.

\section{CFI for position-distribution measurement}

Although the momentum distribution cannot always be resolved, a measurement of the position distribution might be possible. Here the CFI is

$$
F_{C}\left(\hat{S}_{z}, \hat{z}\right)=\sum_{s=a, b} \int d z \frac{\left[\partial_{g} P_{s}(z)\right]^{2}}{P_{S}(z)}
$$

where $P_{s}(z)=\mid\left\langle s|\langle z \mid \Psi(t)\rangle|^{2}\right.$. Figure 2(a) shows this is slightly better than the population-difference measurement, although significantly worse than the momentum measurement. Arguing as before, since the position distribution shifts due to $\hat{z}(t)=$ $\hat{z}(0)+\hat{p}_{z}(0) t / m+\frac{1}{2} g t^{2}$, the additional CFI is $\left(t^{2} / 2\right)^{2} F_{C}^{z}$, where $F_{C}^{z}=\int d z\left[\partial_{z} P(z)\right]^{2} / P(z)$ is the CFI associated with 
resolvable shifts in the position distribution. Since

$$
\begin{aligned}
\operatorname{Var}(z(t))= & \operatorname{Var}(z(0))+\frac{t^{2}}{m^{2}} \operatorname{Var}\left(p_{z}(0)\right) \\
& +\frac{t}{2 m} \operatorname{Cov}\left(p_{z}(0), z(0)\right)
\end{aligned}
$$

and $F_{C}^{z}=1 / \operatorname{Var}(z)$ for Gaussian states, we obtain $\left.F_{C}\left(\hat{S}_{z}, \hat{z}\right)\right|_{2 T_{\pi}}=F_{Q}^{\mathrm{sc}}+8\left(\sigma m T_{\pi}^{2}\right)^{2} /\left[\left(\sigma^{2} m\right)^{2}+\left(2 \hbar T_{\pi}\right)^{2}\right]$ for the initial Gaussian considered in Fig. 2(a), in agreement with numerics.

We can increase $F_{C}\left(\hat{S}_{z}, \hat{z}\right)$ with an initial state that decreases $\operatorname{Var}\left(z\left(2 T_{\pi}\right)\right)$ at the interferometer output. This is not achieved by reducing $\operatorname{Var}(z(0))$, but rather via an initial state with nontrivial correlations between position and momentum such that $\operatorname{Cov}\left(\hat{p}_{z}, \hat{z}\right)$ counteracts the wave packet's ballistic expansion. Figure 2(b) shows the QFI and CFI for initial state $\left\langle z \mid \psi_{0}\right\rangle=e^{-\left(\frac{1}{4}+i\right) z^{2} / 2 \sigma^{2}} /\left[\pi(2 \sigma)^{2}\right]^{1 / 4}$. The imaginary term provides the position-momentum correlations and doubling the spatial width increases the ability of the wave packet to be focused. This initial state could be engineered by applying a harmonic potential for a short duration (compared to motional dynamics), creating phase gradient $\psi(z) \rightarrow \psi(z) e^{-i z^{2} / \sigma_{t}^{2}}$, for constant $\sigma_{t}$ which depends on trap frequency and duration [66]. Then $F_{C}\left(\hat{S}_{z}, \hat{z}\right)$ saturates the QCRB at $T_{1}=T_{2}$, at the cost of reduced $F_{C}\left(\hat{S}_{z}, \hat{p}\right)$.

\section{OPTIMUM MEASUREMENTS}

Since measurements in different bases yield different sensitivities, is there an accessible measurement basis that saturates the QCRB? Our above analysis suggests yes and, depending on the initial state, this optimum basis lies somewhere between position and momentum. We confirm this intuition by revisiting a particle in a gravitational field. We rewrite

$$
|\psi(t)\rangle=\hat{U}_{g}\left|\psi_{0}\right\rangle=\exp \left(-i g \hat{G}_{0}^{\prime}(t)\right)\left|\psi_{0}(t)\right\rangle,
$$

where

$$
\hat{G}_{0}^{\prime}(t)=\hat{U}_{p} \hat{G}_{0}(t) \hat{U}_{p}^{\dagger}=\frac{t}{\hbar}\left(m \hat{z}-\frac{1}{2} \hat{p}_{z} t\right)
$$

$\hat{U}_{p}=\exp \left[-i t \mathbf{p}^{2} /(2 m \hbar)\right]$, and $\left|\psi_{0}(t)\right\rangle=\hat{U}_{p}\left|\psi_{0}\right\rangle$ describes free-particle evolution. We can interpret $\hat{G}_{0}^{\prime}(t)$ as the generator of displacements in $\hat{Q}=c_{1} \hat{z}+c_{2} \hat{p}_{z}$, where the coefficients $c_{i}$ are real and chosen such that $\left[\hat{G}_{0}^{\prime}(t), \hat{Q}\right]=i$. Hence, the probability distribution $|\langle q \mid \psi(t)\rangle|^{2}=\left|\left\langle q-g \mid \psi_{0}(t)\right\rangle\right|^{2}$, where $\hat{Q}|q\rangle=q|q\rangle$. If $\left|\left\langle q \mid \psi_{0}(t)\right\rangle\right|^{2}$ is Gaussian, then measurements of $\hat{Q}$ saturate the QCRB, since $\left[\hat{G}_{0}^{\prime}(t), \hat{Q}\right]=i$ implies

$$
F_{C}(\hat{Q})=\frac{1}{\operatorname{Var}(Q)}=4 \operatorname{Var}\left(G_{0}^{\prime}(t)\right)=F_{Q} .
$$

To measure $\hat{Q}$, we $\operatorname{mix} \hat{z}$ and $\hat{p}_{z}$ by applying the potential $V(z)=\frac{1}{2} m \omega^{2} z^{2}$, since $\hat{z}(t)=\hat{z}(0) \cos \omega t+$ $[\hat{p}(0) / m \omega] \sin \omega t$. Subsequently measuring position yields a combination of position and momentum information. This scheme could be implemented using the following procedure:

(1) At $t=2 T_{\pi}$, apply the unitary $\hat{U}_{s}=|a\rangle\langle a|+$ $|b\rangle\langle b| e^{-i k_{0} \hat{z}}$, which removes any momentum mismatch between the two modes. A state-selective Bragg transition achieves this.

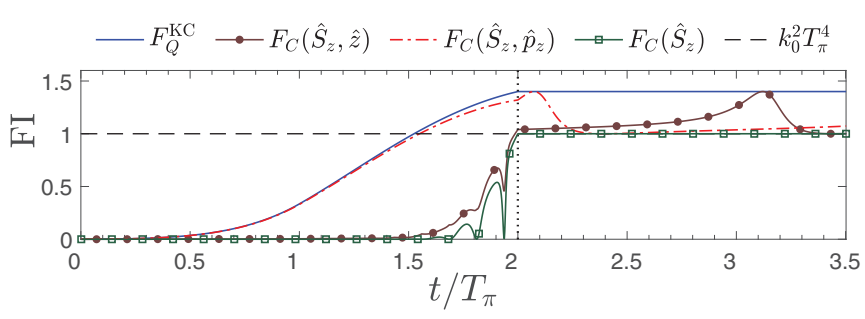

FIG. 3. Fisher information (FI) for $|\Psi(t)\rangle=\hat{U}_{\mathrm{KC}}(t)\left|\Psi_{0}\right\rangle$, where $T_{1}=t$ and $T_{2}=0$ for $t \leqslant T_{\pi}$, otherwise $T_{1}=T_{\pi}$ and $T_{2}=t-T_{\pi}$, with a harmonic potential applied at $t=2 T_{\pi}$ and initial Gaussian motional state $\left\langle z \mid \psi_{0}\right\rangle=\exp \left(-z^{2} / 2 \sigma^{2}\right) /\left(\pi \sigma^{2}\right)^{1 / 4}$. We artificially turned off gravity at $t=2 T_{\pi}$ (which holds $F_{Q}^{\mathrm{KC}}$ constant) to clearly show the effect of harmonic trapping. Specifically, the application of this harmonic potential can be used to saturate the QCRB with either a position-distribution or momentum-distribution measurement. Here $\sigma=10 L, T_{\pi}=100 t_{0}$, and $\omega=3 \pi /\left(2 T_{\pi}\right)$. FI has units $k_{0}^{2} T_{\pi}^{4}$, and length $\left(L=k_{0}^{-1}\right)$ and time $\left(t_{0}=m / \hbar k_{0}^{2}\right)$ units depend on $k_{0}$.

(2) Then apply the potential $V(z)=\frac{1}{2} m \omega^{2}\left(z-z_{0}\right)^{2}$, where $z_{0}=\hbar k_{0} T_{\pi} / m$ is the matter wave's center-of-mass displacement at the interferometer output.

(3) Finally, at some later time, we apply a beam splitter $\hat{U}_{\mathrm{BS}}=\frac{1}{\sqrt{2}}[\hat{1}+(|a\rangle\langle b|-$ H.c. $)]$ immediately before measurement.

Figure 3 shows $F_{C}\left(\hat{S}_{z}, \hat{z}\right)$ and $F_{C}\left(\hat{S}_{z}, \hat{p}_{z}\right)$ for this scheme. Both CFIs oscillate between $F_{Q}^{\text {sc }}$ and the QFI, so a measurement in either the position or momentum basis saturates the QCRB if made at the appropriate time. This improved sensitivity does increase the interferometer time. However, the period of CFI oscillations is negligible compared to $T_{\pi}$ for sufficiently large $\omega$.

\section{IMPROVED INTERFEROMETRY}

In $\mathrm{KC}$ interferometry, the $\pi$ pulse ensures that the wave packets spatially overlap at $t=2 T_{\pi}$. However, Figs. 2 and 3 reveal that spatial overlap is not required for a momentum measurement, making the mirror pulse unnecessary. More interestingly, removing the $\pi$ pulse significantly increases the spatial separation, and therefore the QFI, for the same interrogation time. More precisely, setting $T_{1}=2 T_{\pi}$ and $T_{2}=0$ in Eq. (10) gives $F_{Q}(T)=4 \operatorname{Var}\left(G_{0}(T)\right)+4 k_{0}^{2} T_{\pi}^{4}$, an increase of $3 F_{Q}^{\text {sc }}$ over symmetric KC interferometry.

We numerically solved the Schrödinger equation for the mirrorless Mach-Zehnder (i.e., Ramsey) configuration [Fig. 1(b)]. Figure 4(a) shows that a momentum measurement is always nearly optimal, and at $t=2 T_{\pi}, F_{C}\left(\hat{S}_{z}, \hat{p}_{z}\right) / F_{Q}^{\mathrm{sc}} \approx$ 4.4. Unfortunately, this improved sensitivity has a price: A lack of spatial overlap means that information is encoded in highfrequency interference fringes in the momentum distribution, requiring high-resolution momentum measurements. Following Refs. [67-71], we model imperfect resolution by convolving the momentum distribution at $t=2 T_{\pi}$ with a Gaussian of width $\sigma_{p}$ before constructing $F_{C}\left(\hat{S}_{z}, \hat{p}_{z}\right)$ [Fig. 4(b)]. This imperfect resolution may be due to limitations on the detection system, or other sources of classical noise. The mirrorless configuration is considerably more sensitive to imperfect momentum resolution than KC interferometry, where $F_{C}\left(\hat{S}_{z}, \hat{p}_{z}\right)$ 


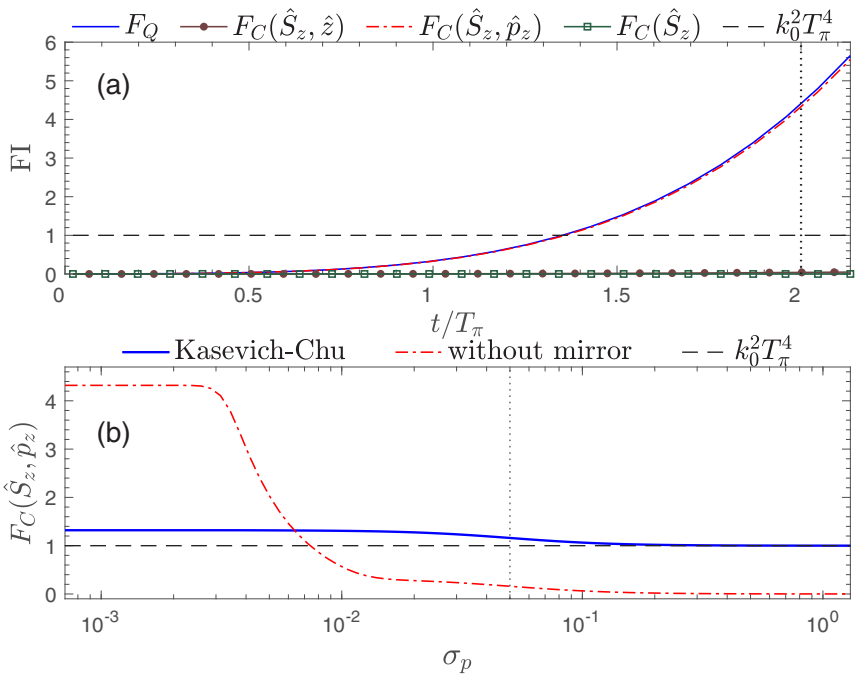

FIG. 4. (a) FI of the mirrorless configuration for the same initial state and parameters as Fig. 2(a). We normalize time by $T_{\pi}=100 t_{0}$ only for comparison with Fig. 2 . Note that $F_{C}\left(\hat{S}_{z}\right)$ and $F_{C}\left(\hat{S}_{z}, \hat{z}\right)$ are almost zero throughout the entire evolution, since there is no spatial overlap of the wave packets and consequently no interference in $P_{s}$ or the position distribution. (b) $F_{C}\left(\hat{S}_{z}, \hat{p}_{z}\right)$ constructed from convolving probabilities with a Gaussian of width $\sigma_{p}$ (units $\hbar k_{0}$ ). The vertical line marks the initial state's momentum width, $\delta p=\hbar / \sqrt{2} \sigma \approx 0.07 \hbar k_{0}$. FI is in units of $k_{0}^{2} T_{\pi}^{4}$.

begins to degrade only when $\sigma_{p}$ is comparable to the initial wave packet's momentum width. Furthermore, in the limit of a "bad" momentum measurement $\left(\sigma_{p} \rightarrow \infty\right)$, the CFI goes to zero, whereas the CFI for KC interferometry approaches $F_{Q}^{\text {sc }}$. Nevertheless, if high-resolution measurements are available (or actively developed), as reported in Ref. [72], for instance, our result suggests that pursuing a mirrorless configuration could yield substantial sensitivity gains.

\section{DISCUSSION AND OUTLOOK}

An important experimental consideration is achieving highresolution momentum measurements. Time-of-flight imaging is a standard technique, where ballistic expansion converts the momentum distribution into a position distribution [73,74]. However, the expansion time needed for sufficient momentum resolution might be significantly longer than the interrogation time, in which case longer interrogation times are a better route to improved sensitivities. Bragg spectroscopy is perhaps a more promising approach [75,76].

Reference [7] reports state-of-the-art gravimetry with a Bose-Einstein condensate (BEC), well described by a pure motional state, and parameters: $\sigma=40 \mu \mathrm{m}, T_{\pi}=130 \mathrm{~ms}$, $k_{0}=1.6 \times 10^{7} \mathrm{~m}^{-1}$, and $\delta p_{z}=0.18 \hbar k_{0}$. We estimate that $4 \operatorname{Var}\left(G_{0}(T)\right)$ is $\sim 7 \%$ of $F_{Q}^{\text {sc }}$, so there is little gain in making optimal measurements [Eq. (11)]. However, $4 \operatorname{Var}\left(G_{0}(T)\right) \sim F_{Q}^{\mathrm{sc}}$ if $\sigma$ or $\delta p_{z}$ were increased by an order of magnitude. This suggests that creating initial (pure) states with large spatial extent, such as quasicontinuous atom lasers [42,77], could yield substantial sensitivity gains. Additionally, compact and/or high-bandwidth devices could benefit from optimal measurements, since shorter interrogation times increase $\operatorname{Var}\left(G_{0}(T)\right)$ relative to $F_{Q}^{\mathrm{sc}}$.

For $\mathrm{KC}$ interferometers with thermal (mixed) states, Eq. (11) is only an upper bound for the QFI [61]. A calculation of $F_{Q}$ and $F_{C}$ for thermal sources gives values substantially greater than $F_{Q}^{\mathrm{sc}}$ [78], in qualitative agreement with our above analysis, showing that current thermal-atom gravimetry is suboptimal. However, the QFI and CFI are also smaller than Eq. (11) for thermal sources, suggesting that BECs possess metrological potential beyond what is possible with thermal sources.

Our approach to evaluating matter-wave interferometry could significantly influence the design of future state-ofthe-art gravimeters. Typical interferometer design assumes a particular form for the measurement signal (e.g., the population difference at the output varies sinusoidally with $g$ ) and looks no further if there is agreement with simple "best case" formulas such as Eq. (1). In contrast, a Fisher analysis gives the full metrological potential of any given dynamical scheme without enforcing such a priori assumptions by simply considering the available data. Our matter-wave gravimetry analysis opens up new routes to improved sensitivity-beyond those few implied by Eq. (1). This includes engineering states with high QFI [i.e., large $\operatorname{Var}\left(G_{0}(T)\right)$ ] and improving information extraction at the interferometer output. Our mirrorless scheme gives a substantial sensitivity boost if high-resolution momentum measurements are available. For Ref. [7], this momentum resolution is $10^{-4} \hbar k_{0}$, achievable by further developing the $2 \times 10^{-4} \hbar k_{0}$ resolution measurement of Ref. [72]. A Fisher analysis could prove beneficial for evaluating other atominterferometer-based sensors which produce a complicated output signal, such as schemes utilizing Kapitza-Dirac scattering [79-84] or propagation in crossed waveguides [85].

\section{ACKNOWLEDGMENTS}

We acknowledge fruitful discussions with John Close, Chris Freier, Paul Griffin, Kyle Hardman, Guglielmo Tino, Nicola Poli, Samuel Nolan, Augusto Smerzi, and Nicholas Robins. M.K. and J.A.D. received funding from UK EPSRC through the Networked Quantum Information Technology (NQIT) Hub, Grant No. EP/M013243/1. S.S.S. received funding from an Australia Awards-Endeavour Research Fellowship, the Dodd-Walls Centre for Photonic and Quantum Technologies, and the Australian Research Council Projects No. DP160104965 and No. DP150100356. S.A.H. was supported by the European Union's Horizon 2020 Research and Innovation Programme under Marie Sklodowska-Curie Grant No. 704672.

\section{APPENDIX A: QFI OF A PARTICLE IN A GRAVITATIONAL FIELD}

Here we give a more detailed derivation of Eq. (5a). Approximating the gravitational field as a linear potential $m g \hat{z}$, the state of the particle after time $T$ is $|\Psi(T)\rangle=\hat{U}_{g}(T)\left|\Psi_{0}\right\rangle$, where

$$
\hat{U}_{g}(T)=\exp \left[-\frac{i T}{\hbar}\left(\frac{\hat{\mathbf{p}}^{2}}{2 m}+m g \hat{z}\right)\right] .
$$


In order to isolate the contribution due to the gravitational field $g$, we make use of the Baker-Campbell-Hausdorff (BCH) lemma:

$$
e^{\hat{X}+\hat{Y}}=e^{\hat{X}} e^{\hat{Y}} e^{-\frac{1}{2}[\hat{X}, \hat{Y}]} e^{\frac{1}{6}(2[\hat{Y},[\hat{X}, \hat{Y}]]+[\hat{X},[\hat{X}, \hat{Y}]])},
$$

where $\hat{X}$ and $\hat{Y}$ are operators satisfying the commutation relations,

$[[[\hat{X}, \hat{Y}], \hat{X}], \hat{X}]=[[[\hat{X}, \hat{Y}], \hat{X}], \hat{Y}]=[[[\hat{X}, \hat{Y}], \hat{Y}], \hat{Y}]=0$.

This is true for $\hat{X}=-\frac{i T}{\hbar} \frac{\hat{\mathbf{p}}^{2}}{2 m}$ and $\hat{Y}=-\frac{i T}{\hbar} m g \hat{z}$, where

$$
\begin{aligned}
{[\hat{X}, \hat{Y}] } & =\frac{i g T^{2}}{\hbar} \hat{p}_{z}, \\
{[\hat{Y},[\hat{X}, \hat{Y}]] } & =\frac{i m g^{2} T^{3}}{\hbar}, \\
{[\hat{X},[\hat{X}, \hat{Y}]] } & =0 .
\end{aligned}
$$

Thus, Eq. (A2) gives

$$
e^{-\frac{i T}{\hbar}\left(\frac{\hat{\mathrm{p}}^{2}}{2 m}+m g \hat{z}\right)}=e^{-\frac{i T}{\hbar} \frac{\hat{\mathrm{p}}^{2}}{2 m}} e^{-\frac{i T}{\hbar} m g \hat{\mathrm{z}}} e^{-\frac{i g T^{2}}{2 \hbar} \hat{p}_{z}} e^{\frac{i m g^{2} T^{3}}{3 \hbar}} .
$$

We use Eq. (A2) again with the choice $\hat{X}=\frac{-i T}{\hbar} m g \hat{z}$ and $\hat{Y}=-\frac{i g T^{2}}{2 \hbar} \hat{p}_{z}$, where $[\hat{X}, \hat{Y}]=-\frac{i m g^{2} T^{3}}{2 \hbar}$, which allows us to combine $\exp [-i(T / \hbar) m g \hat{z}]$ and $\exp \left[-i g T^{2} \hat{p}_{z} /(2 \hbar)\right]$ into a single exponential:

$$
e^{-\frac{i T}{\hbar} m g \hat{z}} e^{-\frac{i g T^{2}}{2 \hbar} \hat{p}_{z}}=e^{-i g \hat{G}_{0}(T)} e^{-\frac{i m g^{2} T^{3}}{4 \hbar}},
$$

where $\hat{G}_{0}(T)=\frac{T}{\hbar}\left(\frac{T}{2} \hat{p}_{z}+m \hat{z}\right)$. Thus, the evolution operator $\hat{U}_{g}(T)$ can be written as

$$
\begin{aligned}
\hat{U}_{g}(T) & =e^{-\frac{i T}{\hbar}\left(\frac{\hat{p}^{2}}{2 m}+m g \hat{z}\right)} \\
& =e^{-i \frac{T}{\hbar} \frac{\hat{p}^{2}}{2 m}} e^{-i g \hat{G}_{0}(T)} e^{i \frac{m g^{2} T^{3}}{12 \hbar}} .
\end{aligned}
$$

We can ignore $\exp \left[i m g^{2} T^{3} /(12 \hbar)\right]$, since this is just a global phase factor, and so the state of the particle after time $T$ is

$$
|\Psi(T)\rangle=e^{-\frac{i T}{\hbar} \frac{\hat{p}^{2}}{2 m}} e^{-i g \hat{G}_{0}(T)}\left|\Psi_{0}\right\rangle .
$$

It is now simple to compute the derivative of $|\Psi(T)\rangle$ with respect to $g$ :

$$
\left|\partial_{g} \Psi(T)\right\rangle=-i e^{-\frac{i T}{\hbar} \frac{\hat{\mathrm{p}}^{2}}{2 m}} \hat{G}_{0}(T) e^{-i g \hat{G}_{0}(T)}\left|\Psi_{0}\right\rangle .
$$

Consequently,

$$
\begin{gathered}
\left\langle\partial_{g} \Psi(T) \mid \partial_{g} \Psi(T)\right\rangle=\left\langle\Psi_{0}\left|\hat{G}_{0}(T)^{2}\right| \Psi_{0}\right\rangle, \\
\left\langle\Psi(T) \mid \partial_{g} \Psi(T)\right\rangle=-i\left\langle\Psi_{0}\left|\hat{G}_{0}(T)\right| \Psi_{0}\right\rangle
\end{gathered}
$$

Substituting these into Eq. (2) gives our final expression for the QFI, Eq. (5a).

\section{APPENDIX B: QFI OF A PARTICLE AFTER KC INTERFEROMETRY}

Here we provide a derivation of Eq. (10). The total evolution of a particle due to $\mathrm{KC}$ interferometry is given by the unitary operator,

$$
\hat{U}_{\mathrm{KC}}=\hat{U}_{\frac{\pi}{2}}^{\phi_{3}} \hat{U}_{g}\left(T_{2}\right) \hat{U}_{\pi}^{\phi_{2}} \hat{U}_{g}\left(T_{1}\right) \hat{U}_{\frac{\pi}{2}}^{\phi_{1}},
$$

where $\hat{U}_{\frac{\pi}{2}}^{\phi}$ and $\hat{U}_{\pi}^{\phi}$ denote $\pi / 2$ (50/50 beam splitting) and $\pi$ (mirror) pulses, respectively, and the evolution due to the gravitational field $\hat{U}_{g}(T)$ was derived above [see Eq. (A7)]. This assumes that the $\pi / 2$ and $\pi$ pulses are instantaneous (strictly, occur on times much shorter than the interrogation times $T_{1}$ and $T_{2}$ ).

To begin, the final $\pi / 2$ pulse does not change the QFI, while the first $\pi / 2$ pulse simply gives a new initial state for the particle [see Eq. (7)]:

$$
\begin{aligned}
\left|\Psi_{0}^{\prime}\right\rangle & =\hat{U}_{\frac{\pi}{2}}^{\phi_{1}}\left|\Psi_{0}\right\rangle \\
& =\frac{1}{\sqrt{2}}\left(\left|\psi_{0}\right\rangle|a\rangle-i e^{i\left(k_{0} \hat{z}-\phi_{1}\right)}\left|\psi_{0}\right\rangle|b\rangle\right),
\end{aligned}
$$

where $\left|\Psi_{0}\right\rangle=|a\rangle\left|\psi_{0}\right\rangle$ and $\phi_{1}$ is the phase of this first laser pulse. Consequently, the QFI can be computed from the product of operators $\hat{U}_{g}\left(T_{2}\right) \hat{U}_{\pi}^{\phi_{2}} \hat{U}_{g}\left(T_{1}\right)$, provided expectations are taken with respect to the state $\left|\Psi_{0}^{\prime}\right\rangle$.

As in Appendix A, our goal is to isolate the $g$ dependence of the evolution. We first consider the product $\hat{U}_{g}\left(T_{2}\right) \hat{U}_{\pi}^{\phi_{2}}$, where [see Eq. (7)]

$$
\hat{U}_{\pi}^{\phi_{2}}=-i\left(e^{-i\left(k_{0} \hat{z}-\phi_{2}\right)}|a\rangle\left\langle b\left|+e^{i\left(k_{0} \hat{z}-\phi_{2}\right)}\right| b\right\rangle\langle a|\right),
$$

and $\phi_{2}$ is the phase of this mirror pulse. The BCH lemma Eq. (A2) implies that

$$
\begin{aligned}
e^{\hat{X}} e^{\hat{Y}} e^{-\frac{1}{2}[\hat{X}, \hat{Y}]} e^{\frac{1}{6}(2[\hat{Y},[\hat{X}, \hat{Y}]]+[\hat{X},[\hat{X}, \hat{Y}]])} \\
=e^{\hat{Y}} e^{\hat{X}} e^{\frac{1}{2}[\hat{X}, \hat{Y}]} e^{-\frac{1}{6}(2[\hat{X},[\hat{X}, \hat{Y}]]+[\hat{Y},[\hat{X}, \hat{Y}]])}
\end{aligned}
$$

The application of Eq. (B4) with $\hat{X}=-i g \hat{G}_{0}\left(T_{2}\right)$ and $\hat{Y}_{ \pm}=$ $\pm i k_{0} \hat{z}$ gives

$$
e^{-i g \hat{G}_{0}\left(T_{2}\right)} e^{ \pm i k_{0} \hat{z}}=e^{ \pm i k_{0} \hat{z}} e^{-i g \hat{G}_{0}\left(T_{2}\right)} e^{\mp i \frac{1}{2} g k_{0} T_{2}^{2}},
$$

where we have used $\left[\hat{X}, \hat{Y}_{ \pm}\right]=\mp i g k_{0} T_{2}^{2} / 2$. Therefore, after neglecting the global phase factor $\exp \left[i m g^{2} T_{2}^{3} /(12 \hbar)\right]$ in $\hat{U}_{g}(T)$ :

$$
\begin{aligned}
& \hat{U}_{g}\left(T_{2}\right) \hat{U}_{\pi}^{\phi_{2}}=-i e^{-\frac{i T_{2}}{\hbar} \frac{\hat{p}^{2}}{2 m}}\left(e^{-i\left(k_{0} \hat{z}-\phi_{2}\right)} e^{-i g \hat{G}_{0}\left(T_{2}\right)} e^{i \frac{1}{2} g k_{0} T_{2}^{2}}|a\rangle\left\langle b\left|+e^{i\left(k_{0} \hat{z}+\phi_{2}\right)} e^{-i g \hat{G}_{0}\left(T_{2}\right)} e^{-i \frac{1}{2} g k_{0} T_{2}^{2}}\right| b\right\rangle\langle a|\right) \\
& =-i e^{-\frac{i T_{2}}{\hbar} \frac{\hat{p}^{2}}{2 m}}\left(e^{-i\left(k_{0} \hat{z}-\phi_{2}\right)} e^{i \frac{1}{2} g k_{0} T_{2}^{2}}|a\rangle\left\langle b\left|+e^{i\left(k_{0} \hat{z}+\phi_{2}\right)} e^{-i \frac{1}{2} g k_{0} T_{2}^{2}}\right| b\right\rangle\langle a|\right) e^{-i g \hat{G}_{0}\left(T_{2}\right)} .
\end{aligned}
$$

Note that $\hat{G}_{0}\left(T_{2}\right)$ acts only on the motional state of the particle and therefore commutes with any operators that act on the internal states $|a\rangle$ and $|b\rangle$.
Now internal states $|a\rangle$ and $|b\rangle$ are the eigenvectors of $\hat{S}_{z}=\frac{1}{2}(|a\rangle\langle a|-| b\rangle\langle b|)$ satisfying $\hat{S}_{z}|a\rangle=\frac{1}{2}|a\rangle$ and $\hat{S}_{z}|b\rangle=$ 
$-\frac{1}{2}|b\rangle$. Therefore, for an arbitrary operator $\hat{O}$ which solely acts on the motional state of the particle:

$$
e^{\hat{O} \hat{S}_{z}}|a\rangle=e^{\frac{1}{2} \hat{o}}|a\rangle, \quad e^{\hat{O} \hat{S}_{z}}|b\rangle=e^{-\frac{1}{2} \hat{o}}|b\rangle .
$$

This allows us to write

$$
\begin{aligned}
\langle a| e^{-i \frac{1}{2} g k_{0} T_{2}^{2}} & =\langle a| e^{-i g k_{0} T_{2}^{2} \hat{S}_{z}}=\langle a| e^{-i g \hat{G}_{e}}, \\
\langle b| e^{i \frac{1}{2} g k_{0} T_{2}^{2}} & =\langle b| e^{-i g k_{0} T_{2}^{2} \hat{S}_{z}}=\langle b| e^{-i g \hat{G}_{e}},
\end{aligned}
$$

where $\hat{G}_{e}=k_{0} T_{2}^{2} \hat{S}_{z}$. Therefore,

$$
\hat{U}_{g}\left(T_{2}\right) \hat{U}_{\pi}^{\phi_{2}}=e^{-\frac{i T_{2}}{\hbar} \frac{\hat{p}^{2}}{2 m}} \hat{U}_{\pi}^{\phi_{2}} e^{-i g \hat{G}_{e}} e^{-i g \hat{G}_{0}\left(T_{2}\right)} .
$$

Next, we again use Eq. (B4) with $\hat{X}=-i g \hat{G}_{0}\left(T_{2}\right)$ and $\hat{Y}=$ $-\frac{i T_{1}}{\hbar} \frac{\hat{\mathbf{p}}^{2}}{2 m}$, where

$$
\begin{aligned}
{[\hat{X}, \hat{Y}] } & =-\frac{i g T_{1} T_{2}}{\hbar} \hat{p}_{z}, \\
{[\hat{X},[\hat{X}, \hat{Y}]] } & =-\frac{i m g^{2} T_{1} T_{2}^{2}}{\hbar},
\end{aligned}
$$

to obtain

$$
\begin{aligned}
& e^{-i g \hat{G}_{0}\left(T_{2}\right)} e^{-i \frac{T_{1}}{\hbar} \frac{\hat{p}^{2}}{2 m}} \\
& =e^{-i \frac{T_{1}}{\hbar} \frac{\hat{p}^{2}}{2 m}} e^{-i g \hat{G}_{0}\left(T_{2}\right)} e^{-i g \frac{T_{1} T_{2}}{\hbar} \hat{p}_{z}} e^{i \frac{m}{2 \hbar} g^{2} T_{1} T_{2}^{2}},
\end{aligned}
$$

and therefore (ignoring the global phase factor $\left.\exp \left[i m g^{2} T_{1} T_{2}^{2} /(2 \hbar)\right]\right)$,

$$
\begin{aligned}
\hat{U}_{g}\left(T_{2}\right) \hat{U}_{\pi}^{\phi_{2}} \hat{U}_{g}\left(T_{1}\right) & =e^{-i \frac{T_{2}}{\hbar} \frac{\hat{\mathrm{p}}^{2}}{2 m}} \hat{U}_{\pi}^{\phi_{2}} e^{-i g \hat{G}_{e}} e^{-i \frac{T_{1}}{\hbar} \frac{\hat{\mathrm{p}}^{2}}{2 m}} e^{-i g \hat{G}_{0}\left(T_{2}\right)} \\
& \times e^{-i g \frac{T_{1} T_{2}}{\hbar} \hat{p}_{z}} e^{-i g \hat{G}_{0}\left(T_{1}\right)}
\end{aligned}
$$

We combine the final three exponentials into one using Eq. (A2):

$$
\begin{aligned}
& \hat{U}_{g}\left(T_{2}\right) \hat{U}_{\pi}^{\phi_{2}} \hat{U}_{g}\left(T_{1}\right) \\
& =e^{-i \frac{T_{2}}{\hbar} \frac{\hat{p}^{2}}{2 m}} \hat{U}_{\pi}^{\phi_{2}} e^{-i \frac{T_{1}}{\hbar} \frac{\hat{p}^{2}}{2 m}} e^{-i g\left(\hat{G}_{0}(T)+\hat{G}_{e}\right)},
\end{aligned}
$$

where $T=T_{1}+T_{2}$ and we have neglected all the global phases produced during the calculation.

Including the first and second $\pi / 2$ pulses (although the second pulse is not needed for calculating the QFI), we arrive at the following simplified expression for the full $\mathrm{KC}$ interferometer evolution:

$$
\hat{U}_{\mathrm{KC}}=\hat{U}_{0} e^{-i g\left(\hat{G}_{0}(T)+\hat{G}_{e}\right)} \hat{U}_{\frac{\pi}{2}}^{\phi_{1}},
$$

where $\hat{U}_{0}=\hat{U}_{\frac{\pi}{2}}^{\phi_{3}} e^{-i \frac{T_{2}}{\hbar} \frac{\hat{p}^{2}}{2 m}} \hat{U}_{\pi}^{\phi_{2}} e^{-i \frac{T_{1}}{\hbar} \frac{p^{2}}{2 m}}$ is independent of $g$. The state of the particle after interrogation time $T$ is therefore

$$
|\Psi(T)\rangle=\hat{U}_{\mathrm{KC}}\left|\Psi_{0}\right\rangle=\hat{U}_{0} e^{-i g\left(\hat{G}_{0}(T)+\hat{G}_{e}\right)}\left|\Psi_{0}^{\prime}\right\rangle,
$$

which is Eq. (8). Taking the derivative with respect to $g$ gives

$$
\begin{aligned}
\left\langle\partial_{g} \Psi(T) \mid \partial_{g} \Psi(T)\right\rangle & =\left\langle\Psi_{0}^{\prime}\left|\left(\hat{G}_{0}(T)+\hat{G}_{e}\right)^{2}\right| \Psi_{0}^{\prime}\right\rangle, \\
\left\langle\Psi(T) \mid \partial_{g} \Psi(T)\right\rangle & =-i\left\langle\Psi_{0}^{\prime}\left|\left(\hat{G}_{0}(T)+\hat{G}_{e}\right)\right| \Psi_{0}^{\prime}\right\rangle .
\end{aligned}
$$

The QFI is therefore

$$
F_{Q}^{\mathrm{KC}}=4 \operatorname{Var}\left(\hat{G}_{0}(T)+\hat{G}_{e}\right),
$$

where the variance is taken with respect to $\left|\Psi_{0}^{\prime}\right\rangle$. We use Eq. (B2) to relate this to expectations taken with respect to the initial state $\left|\Psi_{0}\right\rangle$ :

$$
F_{Q}^{\mathrm{KC}}=4 \operatorname{Var}\left(\hat{G}_{0}(T)\right)+\frac{1}{4} k_{0}^{2}\left(T^{2}-2 T_{2}^{2}\right)^{2},
$$

which is Eq. (10).

\section{APPENDIX C: $\boldsymbol{F}_{C}\left(\hat{S}_{z}\right)$ OF KC INTERFEROMETER}

To calculate the CFI $F_{C}\left(\hat{S}_{z}\right)$ [Eq. (14)], we need to determine expressions for the probabilities $P_{a}(T)$ and $P_{b}(T)$ that the particle is detected in state $|a\rangle$ and $|b\rangle$, respectively, at the interferometer output. This first requires expressing $\hat{U}_{\mathrm{KC}}$ in a more convenient form. To begin, we use Eq. (B4) with $\hat{X}=-i \frac{T_{2}}{\hbar} \frac{\hat{\mathbf{p}}^{2}}{2 m}$ and $\hat{Y}_{ \pm}= \pm i k_{0} \hat{z}$ to obtain

$$
e^{-i \frac{T_{2}}{\hbar} \frac{\hat{p}^{2}}{2 m}} e^{ \pm i k_{0} \hat{z}}=e^{ \pm i k_{0} \hat{z}} e^{-i \frac{T_{2}}{\hbar} \frac{\hat{p}^{2}}{2 m}} e^{\mp i \frac{k_{0} T_{2}}{m} \hat{p}_{z}} e^{-i \frac{\hbar k_{0}^{2} T_{2}}{2 m}},
$$

where we used $\left[\hat{X}, \hat{Y}_{ \pm}\right]=\mp \frac{i k_{0} T_{2}}{m} \hat{p}_{z}$ and $\left[\hat{Y}_{ \pm},\left[\hat{X}, \hat{Y}_{ \pm}\right]\right]=$ $\frac{i \hbar k_{0}^{2} T_{2}}{m}$. This allows us to commute $e^{-i \frac{T_{2}}{\hbar} \frac{\hat{p}^{2}}{2 m}}$ and $\hat{U}_{\pi}^{\phi_{2}}$ :

$$
e^{-i \frac{T_{2}}{\hbar} \frac{\hat{\mathfrak{p}}^{2}}{2 m}} \hat{U}_{\pi}^{\phi_{2}}=\hat{U}_{\pi}^{\phi_{2}} e^{-i \frac{T_{2}}{\hbar} \frac{\hat{\mathrm{p}}^{2}}{2 m}} e^{-2 i \frac{k_{0} T_{2}}{m} \hat{p}_{z} \hat{S}_{z}} e^{-i \frac{\hbar k_{0}^{2} T_{2}}{2 m}}
$$

where we have again used Eq. (B7). Neglecting the global phase factor $\exp \left[-i \hbar k_{0}^{2} T_{2} /(2 m)\right]$, we can therefore write Eq. (B14) in the convenient form,

$$
\hat{U}_{\mathrm{KC}}=\hat{U}_{\mathrm{int}} \hat{U}_{\mathrm{ext}} \hat{U}_{\frac{\pi}{2}}^{\phi_{1}},
$$

where

$$
\begin{aligned}
& \hat{U}_{\mathrm{int}} \equiv \hat{U}_{\frac{\pi}{2}}^{\phi_{3}} \hat{U}_{\pi}^{\phi_{2}} e^{-2 i \frac{k_{0} T_{2}}{m} \hat{p}_{z} \hat{S}_{z}} e^{-i g \hat{G}_{e}}, \\
& \hat{U}_{\mathrm{ext}} \equiv e^{-i \frac{T}{\hbar} \frac{\hat{p}^{2}}{2 m}} e^{-i g \hat{G}_{0}(T)} .
\end{aligned}
$$

$\hat{U}_{\text {ext }}$ only acts on the external (i.e., motional) degrees of freedom, whereas $\hat{U}_{\text {int }}$ acts on both the internal and motional degrees of freedom. Note that $\hat{U}_{\text {int }}$ and $\hat{U}_{\text {ext }}$ do not commute.

The state of the particle at the output of the interferometer after interrogation time $T$ is therefore

$$
\begin{aligned}
|\Psi(T)\rangle & =\hat{U}_{\mathrm{int}} \hat{U}_{\mathrm{ext}} \hat{U}_{\frac{\pi}{2}}^{\phi_{1}}\left|\Psi_{0}\right\rangle \\
& =\frac{1}{\sqrt{2}}\left(\hat{U}_{\mathrm{int}}|a\rangle \hat{U}_{\mathrm{ext}}\left|\psi_{0}\right\rangle-i \hat{U}_{\mathrm{int}}|b\rangle \hat{U}_{\mathrm{ext}} e^{i\left(k_{0} \hat{z}-\phi_{1}\right)}\left|\psi_{0}\right\rangle\right) .
\end{aligned}
$$

From Eq. (7) we get

$$
\begin{aligned}
\hat{U}_{\frac{\pi}{2}}^{\phi_{3}} \hat{U}_{\pi}^{\phi_{2}} & =-\frac{1}{\sqrt{2}}\left(e^{-i\left(\phi_{2}-\phi_{3}\right)}|a\rangle\left\langle a\left|+e^{i\left(\phi_{2}-\phi_{3}\right)}\right| b\right\rangle\langle b|\right) \\
& -\frac{i}{\sqrt{2}}\left(e^{-i\left(k_{0} \hat{z}-\phi_{2}\right)}|a\rangle\left\langle b\left|+e^{i\left(k_{0} \hat{z}-\phi_{2}\right)}\right| b\right\rangle\langle a|\right),
\end{aligned}
$$

where $\phi_{2}$ and $\phi_{3}$ are the phases of the second and the third laser pulses, respectively. Using this and Eq. (B7), we 
obtain

$$
\begin{aligned}
\hat{U}_{\text {int }}|a\rangle= & -\frac{1}{\sqrt{2}}\left[e^{-i\left(\phi_{2}-\phi_{3}\right)}|a\rangle+i e^{i\left(k_{0} \hat{z}-\phi_{2}\right)}|b\rangle\right] \\
& \times e^{-i \frac{k_{0} T_{2}}{m} \hat{p}_{z}} e^{-i g \frac{k_{0} T_{2}^{2}}{2}}
\end{aligned}
$$

$$
\begin{aligned}
\hat{U}_{\text {int }}|b\rangle= & -\frac{1}{\sqrt{2}}\left[e^{i\left(\phi_{2}-\phi_{3}\right)}|b\rangle+i e^{-i\left(k_{0} \hat{z}-\phi_{2}\right)}|a\rangle\right] \\
& \times e^{i \frac{k_{0} T_{2}}{m} \hat{p}_{z}} e^{i \frac{k_{0} T_{2}^{2}}{2}} .
\end{aligned}
$$

(C8a)

Substituting Eqs. (C8) into Eq. (C6) gives

$$
\begin{aligned}
& |\Psi(T)\rangle=-\frac{1}{2}\left[\left(e^{-i\left(\phi_{2}-\phi_{3}\right)} e^{-i \frac{k_{0} T_{2}}{m} \hat{p}_{z}} e^{-i g} \frac{k_{0} T_{2}^{2}}{2} \hat{U}_{\mathrm{ext}}\left|\psi_{0}\right\rangle+e^{-i\left(k_{0} \hat{z}-\phi_{2}\right)} e^{i \frac{k_{0} T_{2}}{m} \hat{p}_{z}} e^{\frac{i}{2} g k_{0} T_{2}^{2}} \hat{U}_{\mathrm{ext}} e^{i\left(k_{0} \hat{z}-\phi_{1}\right)}\left|\psi_{0}\right\rangle\right)|a\rangle\right. \\
& \left.+i\left(e^{i\left(k_{0} \hat{z}-\phi_{2}\right)} e^{-i \frac{k_{0} T_{2}}{m} \hat{p}_{z}} e^{-i g^{\frac{k_{0} T_{2}^{2}}{2}}} \hat{U}_{\mathrm{ext}}\left|\psi_{0}\right\rangle-e^{i\left(\phi_{2}-\phi_{3}\right)} e^{i k_{0} T_{2}} \frac{\hat{p}_{z}}{e^{\frac{i}{2} g k_{0} T_{2}^{2}}} \hat{U}_{\mathrm{ext}} e^{i\left(k_{0} \hat{\mathrm{z}}-\phi_{1}\right)}\left|\psi_{0}\right\rangle\right)|b\rangle\right] .
\end{aligned}
$$

Defining $\left|\Psi_{a}(T)\right\rangle \equiv\langle a \mid \Psi(T)\rangle$, the probability of finding the particle in the internal state $|a\rangle$ at the output port of the interferometer is

$$
\begin{aligned}
P_{a}(T) & =\left\langle\Psi_{a}(T) \mid \Psi_{a}(T)\right\rangle \\
& =\frac{1}{2}\left[1+\frac{1}{2}\left(e^{i\left(g k_{0} T_{2}^{2}-\Phi\right)}\left\langle\psi_{0}|\hat{Q}| \psi_{0}\right\rangle+\text { H.c. }\right)\right],
\end{aligned}
$$

where $\Phi \equiv \phi_{1}-2 \phi_{2}+\phi_{3}$ and

$$
\begin{aligned}
\hat{Q} \equiv & e^{i g \hat{G}_{0}(T)} e^{i \frac{T}{\hbar} \frac{\hat{p}^{2}}{2 m}} e^{i \frac{k_{0} T_{2}}{m} \hat{p}_{z}} e^{-i k_{0} \hat{z}} \\
& \times e^{i \frac{k_{0} T_{2}}{m} \hat{p}_{z}} e^{-i \frac{T}{\hbar} \frac{\hat{p}^{2}}{2 m}} e^{-i g \hat{G}_{0}(T)} e^{i k_{0} \hat{z}} \\
= & e^{i \frac{\hbar k_{0}^{2}}{2 m}\left(T_{2}-T_{1}\right)} e^{-i g k_{0} T\left(T_{2}-T_{1}\right)} e^{-i g \frac{k_{0} T^{2}}{2}} e^{i \frac{k_{0}}{m}\left(T_{2}-T_{1}\right) \hat{p}_{z}} .
\end{aligned}
$$

This final simplification follows from repeated application of Eq. (B4), and allows us to express the probability as

$$
\begin{aligned}
P_{a}(T)= & \frac{1}{2}\left[1+\frac{1}{2}\left(e^{-i \Phi} e^{i \frac{\hbar k_{0}^{2}}{2 m}\left(T_{2}-T_{1}\right)} e^{-i g k_{0}\left(\frac{T^{2}}{2}-T_{1}^{2}\right)}\right.\right. \\
& \left.\left.\times\left\langle\psi_{0}\left|e^{i \frac{k_{0}}{m}\left(T_{2}-T_{1}\right) \hat{p}_{z}}\right| \psi_{0}\right\rangle+\text { H.c. }\right)\right] .
\end{aligned}
$$

If we choose the phases of our laser pulses such that $\phi_{1}=$ $\phi_{2}=0, \phi_{3}=\pi / 2$, thereby operating at the point of maximum sensitivity, we can express the probabilities in the following way:

$$
\begin{aligned}
& P_{a}(T)=\frac{1}{2}\left[1-\frac{i}{2}\left(\mathcal{C} e^{i\left(\phi_{f}-\phi_{g}\right)}-\mathcal{C}^{*} e^{-i\left(\phi_{f}-\phi_{g}\right)}\right)\right], \\
& P_{b}(T)=\frac{1}{2}\left[1+\frac{i}{2}\left(\mathcal{C} e^{i\left(\phi_{f}-\phi_{g}\right)}-\mathcal{C}^{*} e^{-i\left(\phi_{f}-\phi_{g}\right)}\right)\right],
\end{aligned}
$$

where

$$
\begin{gathered}
\phi_{f} \equiv \frac{\hbar k_{0}^{2}}{2 m}\left(T_{2}-T_{1}\right), \\
\phi_{g} \equiv k_{0} g\left(\frac{T^{2}}{2}-T_{1}^{2}\right), \\
\mathcal{C} \equiv\left\langle\psi_{0}\left|e^{i \frac{k_{0}}{m}\left(T_{2}-T_{1}\right) \hat{p}_{z}}\right| \psi_{0}\right\rangle .
\end{gathered}
$$

$\phi_{f}$ represents the phase difference due to the nonsymmetrical free evolution of the wave packets in the two arms of the interferometer, while $\phi_{g}$ is the phase difference due to gravity. Expressing $\mathcal{C}=|\mathcal{C}| e^{i \vartheta}$ allows us to write Eq. (C13) in the simplified form of Eq. (13). Here $|\mathcal{C}|$ is interpreted as a fringe contrast and $\alpha=\phi_{f}-\phi_{g}+\vartheta$ denotes the total phase shift.

If we measure the population difference of the two internal states $\hat{S}_{z}$ at the output of the interferometer, the CFI is given by

$$
F_{C}\left(\hat{S}_{z}\right)=\sum_{j=a, b} \frac{\left(\partial_{g} P_{j}\right)^{2}}{P_{j}}=\frac{\left(\partial_{g} P_{a}\right)^{2}}{P_{a} P_{b}},
$$

where the last equality follows from the relation $P_{a}+P_{b}=$ $1 \Rightarrow \partial_{g} P_{a}=-\partial_{g} P_{b}$. Noting that

$$
\begin{gathered}
P_{a}(T) P_{b}(T)=\frac{1}{4}\left(1-|\mathcal{C}|^{2} \sin ^{2} \alpha\right), \\
\partial_{g} P_{a}(T)=-\frac{1}{2}|\mathcal{C}| k_{0}\left(\frac{T^{2}}{2}-T_{1}^{2}\right) \cos \alpha,
\end{gathered}
$$

we arrive at Eq. (14).

\section{APPENDIX D: BEAM SPLITTER TRANSFORMATION: DERIVATION OF EQ. (7).}

A Raman beam splitter is typically modeled by the Hamiltonian,

$$
\hat{H}_{\mathrm{BS}}=\frac{\hat{\mathbf{p}}^{2}}{2 m}-\hbar \delta|b\rangle\langle b|+\frac{\hbar \Omega}{2}\left(|b\rangle\langle a| e^{i\left(k_{0} \hat{z}-\phi\right)}+\text { H.c. }\right),
$$

where $\delta$ is the two-photon detuning and $\Omega=\Omega_{1} \Omega_{2} / \Delta$ is the effective two-photon Rabi frequency, which depends on the single-photon Rabi frequencies $\Omega_{1,2}$ and the single-photon detuning $\Delta[86,87]$. The two-photon detuning is typically set to the two-photon resonance condition $\delta=\hbar k_{0}^{2} /(2 m)$. Evolution under this Hamiltonian for a duration $\Delta t$ is given by the unitary 


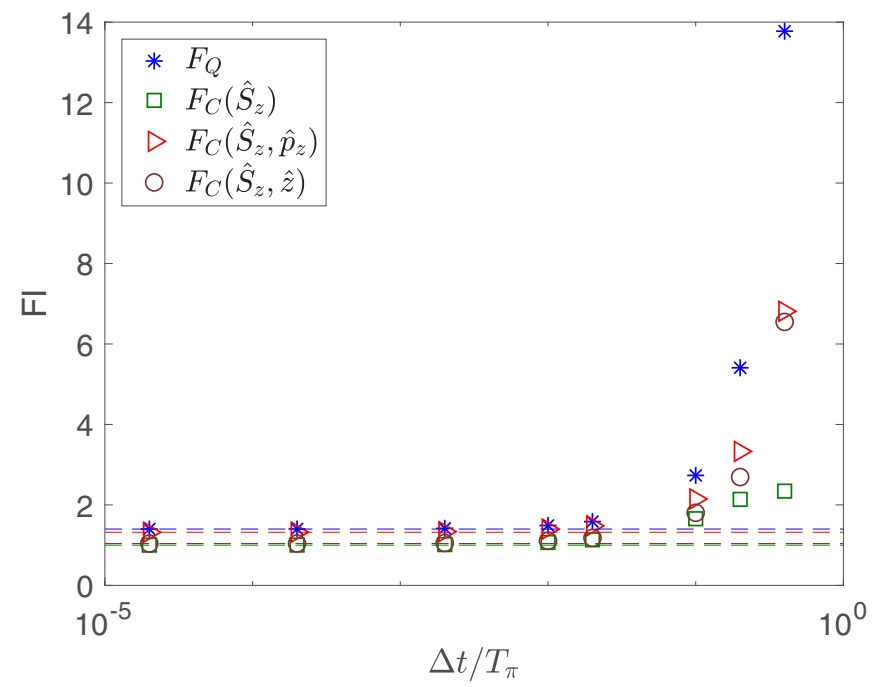

FIG. 5. (a) QFI and CFI computed using Eq. (D1) rather than Eq. (D3) as a function of $\Delta t$. Provided $\Delta t / T_{\pi} \ll 1$, Eq. (D3) (shown by dashed lines of the appropriate color) is an excellent approximation to the true dynamics. Fisher information is presented in units of $k_{0}^{2} T_{\pi}^{4}$.

time-evolution operator,

$$
\begin{aligned}
U_{\theta}^{\phi} & =\exp \left[\frac{-i \Delta t}{\hbar} \hat{H}_{\mathrm{BS}}\right] \\
& =e^{-i\left(\frac{\hat{\mathrm{p}}^{2}}{2 m \hbar}-\frac{\hbar k_{0}^{2}}{2 m}|b\rangle\langle b|\right) \frac{\theta}{\Omega}-i \frac{\theta}{2}\left(|b\rangle\langle a| e^{i\left(k_{0} \hat{\mathrm{z}}-\phi\right)}+\text { H.c. }\right)},
\end{aligned}
$$

where we have defined $\theta=\Omega \Delta t$. If $\hbar \Omega$ is significantly greater than the spread in kinetic energy of the initial state, we can ignore the first term and obtain

$$
\begin{aligned}
U_{\theta}^{\phi} & =\exp \left[-i \frac{\theta}{2}\left(|b\rangle\langle a| e^{i\left(k_{0} \hat{z}-\phi\right)}+\text { H.c. }\right)\right] \\
& =\hat{1} \cos \left(\frac{\theta}{2}\right)-i\left(|b\rangle\langle a| e^{i\left(k_{0} \hat{z}-\phi\right)}+\text { H.c. }\right) \sin \left(\frac{\theta}{2}\right),
\end{aligned}
$$

which is Eq. (7).

Figure 5 shows the QFI and CFI when the evolution due to the beam splitter and mirror pulses is treated as Schrödinger evolution under Hamiltonian Eq. (D1). This evolution was solved numerically for different values of $\Delta t$. We used the same initial state as Fig. 2(a). We set $\Omega$ such that $\Omega \Delta t=\pi / 2$ for the two beam splitter pulses, and the duration of the interaction was doubled for the mirror pulse, resulting in $\Omega(2 \Delta t)=\pi$. We find excellent agreement with the ideal beam splitter case as long as $\Delta t \ll T_{\pi}$. In the regime $\Delta t \sim T_{\pi}$, there is significant motional dynamics during the beam splitter period, and our approximation is no longer valid. For example, for the maximum value of $\Delta t$ simulated $\left(\Delta t=0.4 T_{\pi}\right)$, the total interferometer sequence time, which is the time from the commencement of the first beam splitter to the conclusion of the second beam splitter, is $3.6 T_{\pi}$ (compared to $2 T_{\pi}$ for instantaneous beam splitters). For typical experiments, such as Ref. [7], $\Delta t / T_{\pi} \sim 10^{-4}$.
[1] A. Peters, K. Y. Chung, and S. Chu, Measurement of gravitational acceleration by dropping atoms, Nature (London) 400, 849 (1999).

[2] A. Peters, K. Y. Chung, and S. Chu, High-precision gravity measurements using atom interferometry, Metrologia 38, 25 (2001).

[3] Z.-K. Hu, B.-L. Sun, X.-C. Duan, M.-K. Zhou, L.-L. Chen, S. Zhan, Q.-Z. Zhang, and J. Luo, Demonstration of an ultrahighsensitivity atom-interferometry absolute gravimeter, Phys. Rev. A 88, 043610 (2013).

[4] M. Hauth, C. Freier, V. Schkolnik, A. Senger, M. Schmidt, and A. Peters, First gravity measurements using the mobile atom interferometer gain, Appl. Phys. B 113, 49 (2013).

[5] P. A. Altin, M. T. Johnsson, V. Negnevitsky, G. R. Dennis, R. P. Anderson, J. E. Debs, S. S. Szigeti, K. S. Hardman, S. Bennetts, G. D. McDonald, L. D. Turner, J. D. Close, and N. P. Robins, Precision atomic gravimeter based on bragg diffraction, New J. Phys. 15, 023009 (2013).

[6] C. Freier, M. Hauth, V. Schkolnik, B. Leykauf, M. Schilling, H. Wziontek, H.-G. Scherneck, J. Müller, and A. Peters, Mobile quantum gravity sensor with unprecedented stability, J. Phys.: Conf. Ser. 723, 012050 (2016).

[7] K. S. Hardman, P. J. Everitt, G. D. McDonald, P. Manju, P. B. Wigley, M. A. Sooriyabandara, C. C. N. Kuhn, J. E. Debs, J. D. Close, and N. P. Robins, Simultaneous Precision Gravimetry and Magnetic Gradiometry with a Bose-Einstein Condensate: A High Precision, Quantum Sensor, Phys. Rev. Lett. 117, 138501 (2016).
[8] M. J. Snadden, J. M. McGuirk, P. Bouyer, K. G. Haritos, and M. A. Kasevich, Measurement of the Earth's Gravity Gradient with an Atom Interferometer-Based Gravity Gradiometer, Phys. Rev. Lett. 81, 971 (1998).

[9] G. Stern, B. Battelier, R. Geiger, G. Varoquaux, A. Villing, F. Moron, O. Carraz, N. Zahzam, Y. Bidel, W. Chaibi, F. Pereira Dos Santos, A. Bresson, A. Landragin, and P. Bouyer, Lightpulse atom interferometry in microgravity, Eur. Phys. J. D 53, 353 (2009).

[10] F. Sorrentino, Q. Bodart, L. Cacciapuoti, Y.-H. Lien, M. Prevedelli, G. Rosi, L. Salvi, and G. M. Tino, Sensitivity limits of a Raman atom interferometer as a gravity gradiometer, Phys. Rev. A 89, 023607 (2014).

[11] G. W. Biedermann, X. Wu, L. Deslauriers, S. Roy, C. Mahadeswaraswamy, and M. A. Kasevich, Testing gravity with cold-atom interferometers, Phys. Rev. A 91, 033629 (2015).

[12] G. D’Amico, F. Borselli, L. Cacciapuoti, M. Prevedelli, G. Rosi, F. Sorrentino, and G. M. Tino, Bragg interferometer for gravity gradient measurements, Phys. Rev. A 93, 063628 (2016).

[13] P. Asenbaum, C. Overstreet, T. Kovachy, D. D. Brown, J. M. Hogan, and M. A. Kasevich, Phase Shift in an Atom Interferometer Due to Spacetime Curvature Across Its Wave Function, Phys. Rev. Lett. 118, 183602 (2017).

[14] Y. Bidel, N. Zahzam, C. Blanchard, A. Bonnin, M. Cadoret, A. Bresson, D. Rouxel, and M. F. Lequentrec-Lalancette, Absolute marine gravimetry with matter-wave interferometry, Nat. Commun. 9, 627 (2018). 
[15] J. A. Richeson, Gravity Gradiometer Aided Inertial Navigation within Non-GNSS Environments, Ph.D. thesis, University of Maryland, 2008 .

[16] M. I. Evstifeev, The state of the art in the development of onboard gravity gradiometers, Gyroscopy and Navigation 8, 68 (2017).

[17] S. Fray, C. A. Diez, T. W. Hänsch, and M. Weitz, Atomic Interferometer with Amplitude Gratings of Light and Its Applications to Atom Based Tests of the Equivalence Principle, Phys. Rev. Lett. 93, 240404 (2004).

[18] S. Dimopoulos, P. W. Graham, J. M. Hogan, and M. A. Kasevich, Testing General Relativity with Atom Interferometry, Phys. Rev. Lett. 98, 111102 (2007).

[19] D. Schlippert, J. Hartwig, H. Albers, L. L. Richardson, C. Schubert, A. Roura, W. P. Schleich, W. Ertmer, and E. M. Rasel, Quantum Test of the Universality of Free Fall, Phys. Rev. Lett. 112, 203002 (2014).

[20] G. Amelino-Camelia, C. Lämmerzahl, F. Mercati, and G. M. Tino, Constraining the Energy-Momentum Dispersion Relation with Planck-Scale Sensitivity Using Cold Atoms, Phys. Rev. Lett. 103, 171302 (2009).

[21] D. Gao and M. Zhan, Constraining the generalized uncertainty principle with cold atoms, Phys. Rev. A 94, 013607 (2016).

[22] Ch. J. Bordé, Atomic interferometry with internal state labelling, Phys. Lett. A 140, 10 (1989).

[23] M. Kasevich and S. Chu, Atomic Interferometry Using Stimulated Raman Transitions, Phys. Rev. Lett. 67, 181 (1991).

[24] M. Kasevich and S. Chu, Measurement of the gravitational acceleration of an atom with a light-pulse atom interferometer, Appl. Phys. B 54, 321 (1992).

[25] P. Storey and C. Cohen-Tannoudji, The Feynman path integral approach to atomic interferometry. A tutorial, J. Phys. II 4, 1999 (1994).

[26] W. P. Schleich, D. M. Greenberger, and E. M. Rasel, Redshift Controversy in Atom Interferometry: Representation Dependence of the Origin of Phase Shift, Phys. Rev. Lett. 110, 010401 (2013).

[27] W. P. Schleich, D. M Greenberger, and E. M Rasel, A representation-free description of the Kasevich-Chu interferometer: A resolution of the redshift controversy, New J. Phys. 15, 013007 (2013).

[28] H. Müller, S.-W. Chiow, Q. Long, S. Herrmann, and S. $\mathrm{Chu}$, Atom Interferometry with Up to 24-Photon-MomentumTransfer Beam Splitters, Phys. Rev. Lett. 100, 180405 (2008).

[29] P. Cladé, S. Guellati-Khélifa, F. Nez, and F. Biraben, Large Momentum Beam Splitter Using Bloch Oscillations, Phys. Rev. Lett. 102, 240402 (2009).

[30] S.-W. Chiow, T. Kovachy, H.-C. Chien, and M. A. Kasevich, $102 \hbar k$ Large Area Atom Interferometers, Phys. Rev. Lett. 107, 130403 (2011).

[31] G. D. McDonald, C. C. N. Kuhn, S. Bennetts, J. E. Debs, K. S. Hardman, M. Johnsson, J. D. Close, and N. P. Robins, $80 \hbar k$ momentum separation with Bloch oscillations in an optically guided atom interferometer, Phys. Rev. A 88, 053620 (2013).

[32] T. Mazzoni, X. Zhang, R. Del Aguila, L. Salvi, N. Poli, and G. M. Tino, Large-momentum-transfer Bragg interferometer with strontium atoms, Phys. Rev. A 92, 053619 (2015).

[33] C. Gross, T. Zibold, E. Nicklas, J. Estève, and M. K. Oberthaler, Nonlinear atom interferometer surpasses classical precision limit, Nature (London) 464, 1165 (2010).
[34] B. Lücke, M. Scherer, J. Kruse, L. Pezzé, F. Deuretzbacher, P. Hyllus, O. Topic, J. Peise, W. Ertmer, J. Arlt, L. Santos, A. Smerzi, and C. Klempt, Twin matter waves for interferometry beyond the classical limit, Science 334, 773 (2011).

[35] D. Linnemann, H. Strobel, W. Muessel, J. Schulz, R. J. LewisSwan, K. V. Kheruntsyan, and M. K. Oberthaler, QuantumEnhanced Sensing Based on Time Reversal of Nonlinear Dynamics, Phys. Rev. Lett. 117, 013001 (2016).

[36] O. Hosten, N. J. Engelsen, R. Krishnakumar, and M. A. Kasevich, Measurement noise 100 times lower than the quantumprojection limit using entangled atoms, Nature (London) 529, 505 (2016).

[37] G. Colangelo, F. Martin Ciurana, G. Puentes, M. W. Mitchell, and R. J. Sewell, Entanglement-Enhanced Phase Estimation without Prior Phase Information, Phys. Rev. Lett. 118, 233603 (2017).

[38] J. E. Debs, P. A. Altin, T. H. Barter, D. Döring, G. R. Dennis, G. McDonald, R. P. Anderson, J. D. Close, and N. P. Robins, Cold-atom gravimetry with a Bose-Einstein condensate, Phys. Rev. A 84, 033610 (2011).

[39] A. Louchet-Chauvet, T. Farah, Q. Bodart, A. Clairon, A. Landragin, S. Merlet, and F. Pereira Dos Santos, The influence of transverse motion within an atomic gravimeter, New J. Phys. 13, 065025 (2011).

[40] S. S. Szigeti, J. E. Debs, J. J. Hope, N. P. Robins, and J. D. Close, Why momentum width matters for atom interferometry with Bragg pulses, New J. Phys. 14, 023009 (2012).

[41] K. S. Hardman, C. C. N. Kuhn, G. D. McDonald, J. E. Debs, S. Bennetts, J. D. Close, and N. P. Robins, Role of source coherence in atom interferometery, Phys. Rev. A 89, 023626 (2014).

[42] N. P. Robins, P. A. Altin, J. E. Debs, and J. D. Close, Atom lasers: Production, properties and prospects for precision inertial measurement, Phys. Rep. 529, 265 (2013).

[43] S. S. Szigeti, M. R. Hush, A. R. R. Carvalho, and J. J. Hope, Continuous measurement feedback control of a Bose-Einstein condensate using phase-contrast imaging, Phys. Rev. A 80, 013614 (2009).

[44] S. S. Szigeti, M. R. Hush, A. R. R. Carvalho, and J. J. Hope, Feedback control of an interacting Bose-Einstein condensate using phase-contrast imaging, Phys. Rev. A 82, 043632 (2010).

[45] M. R. Hush, S. S. Szigeti, A. R. R. Carvalho, and J. J. Hope, Controlling spontaneous-emission noise in measurement-based feedback cooling of a Bose-Einstein condensate, New J. Phys. 15, 113060 (2013).

[46] P. A. Altin, G. McDonald, D. Doring, J. E. Debs, T. H. Barter, J. D. Close, N. P. Robins, S. A. Haine, T. M. Hanna, and R. P. Anderson, Optically trapped atom interferometry using the clock transition of large $87 \mathrm{rb}$ Bose-Einstein condensates, New J. Phys. 13, 065020 (2011).

[47] S. A. Haine and A. J. Ferris, Surpassing the standard quantum limit in an atom interferometer with four-mode entanglement produced from four-wave mixing, Phys. Rev. A 84, 043624 (2011).

[48] S. A. Haine, Information-Recycling Beam Splitters for Quantum Enhanced Atom Interferometry, Phys. Rev. Lett. 110, 053002 (2013).

[49] S. A. Haine, J. Lau, R. P. Anderson, and M. T. Johnsson, Selfinduced spatial dynamics to enhance spin squeezing via one-axis twisting in a two-component Bose-Einstein condensate, Phys. Rev. A 90, 023613 (2014). 
[50] S. S. Szigeti, B. Tonekaboni, W. Y. S. Lau, S. N. Hood, and S. A. Haine, Squeezed-light-enhanced atom interferometry below the standard quantum limit, Phys. Rev. A 90, 063630 (2014).

[51] B. Tonekaboni, S. A. Haine, and S. S. Szigeti, Heisenberglimited metrology with a squeezed vacuum state, three-mode mixing, and information recycling, Phys. Rev. A 91, 033616 (2015).

[52] S. A. Haine, S. S. Szigeti, M. D. Lang, and C. M. Caves, Heisenberg-limited metrology with information recycling, Phys. Rev. A 91, 041802 (2015).

[53] S. A. Haine and S. S. Szigeti, Quantum metrology with mixed states: When recovering lost information is better than never losing it, Phys. Rev. A 92, 032317 (2015).

[54] S. P. Nolan, J. Sabbatini, M. W. J. Bromley, M. J. Davis, and S. A. Haine, Quantum enhanced measurement of rotations with a spin-1 Bose-Einstein condensate in a ring trap, Phys. Rev. A 93, 023616 (2016).

[55] S. A. Haine and W. Y. S. Lau, Generation of atom-light entanglement in an optical cavity for quantum enhanced atom interferometry, Phys. Rev. A 93, 023607 (2016).

[56] S. S. Szigeti, R. J. Lewis-Swan, and S. A. Haine, Pumped-Up su(1,1) Interferometry, Phys. Rev. Lett. 118, 150401 (2017).

[57] S. A Haine, Quantum noise in bright soliton matterwave interferometry, New J. Phys. 20, 033009 (2018).

[58] S.-Y. Lan, P.-C. Kuan, B. Estey, P. Haslinger, and H. Müller, Influence of the Coriolis Force in Atom Interferometry, Phys. Rev. Lett. 108, 090402 (2012).

[59] V. Schkolnik, B. Leykauf, M. Hauth, C. Freier, and A. Peters, The effect of wavefront aberrations in atom interferometry, Appl. Phys. B 120, 311 (2015).

[60] S. L. Braunstein and C. M. Caves, Statistical Distance and The Geometry of Quantum States, Phys. Rev. Lett. 72, 3439 (1994).

[61] R. Demkowicz-Dobrzański, M. Jarzyna, and J. Kołodyński, Quantum limits in optical interferometry, Prog. Opt. 60, 345 (2015).

[62] G. Tóth and I. Apellaniz, Quantum metrology from a quantum information science perspective, J. Phys. A: Math. Theor. 47, 424006 (2014).

[63] S. A. Haine, Mean-Field Dynamics and Fisher Information in Matter Wave Interferometry, Phys. Rev. Lett. 116, 230404 (2016).

[64] S. Qvarfort, A. Serafini, P. Barker, and S. Bose, Gravimetry through non-linear optomechanics, arXiv:1706.9131.

[65] M. R. Matthews, B. P. Anderson, P. C. Haljan, D. S. Hall, M. J. Holland, J. E. Williams, C. E. Wieman, and E. A. Cornell, Watching a Superfluid Untwist Itself: Recurrence of Rabi Oscillations in a Bose-Einstein Condensate, Phys. Rev. Lett. 83, 3358 (1999).

[66] H. Ammann and N. Christensen, Delta Kick Cooling: A New Method for Cooling Atoms, Phys. Rev. Lett. 78, 2088 (1997).

[67] L. Pezzé and A. Smerzi, Ultrasensitive Two-Mode Interferometry with Single-Mode Number Squeezing, Phys. Rev. Lett. 110, 163604 (2013).

[68] M. Gabbrielli, L. Pezzè, and A. Smerzi, Spin-Mixing Interferometry with Bose-Einstein Condensates, Phys. Rev. Lett. 115, 163002 (2015).

[69] S. P. Nolan, S. S. Szigeti, and S. A. Haine, Optimal and Robust Quantum Metrology Using Interaction-Based Readouts, Phys. Rev. Lett. 119, 193601 (2017).
[70] S. S. Mirkhalaf, S. P. Nolan, and S. A. Haine, Robustifying twistand-turn entanglement with interaction-based readout, Phys. Rev. A 97, 053618 (2018).

[71] S. A. Haine, Using interaction-based readouts to approach the ultimate limit of detection noise robustness for quantum-enhanced metrology in collective spin systems, arXiv:1806.0057.

[72] I. Gotlibovych, T. F. Schmidutz, A. L. Gaunt, N. Navon, R. P. Smith, and Z. Hadzibabic, Observing properties of an interacting homogeneous Bose-Einstein condensate: Heisenberg-limited momentum spread, interaction energy, and free-expansion dynamics, Phys. Rev. A 89, 061604 (2014).

[73] W. Ketterle, D. S. Durfee, and D. M. Stamper-Kurn, Making, Probing and Understanding Bose-Einstein Condensates, in Proceedings of the International School of Physics "Enrico Fermi," Vol. 140, edited by M. Inguscio, S. Stringari, and C. E. Wieman (IOS Press, Amsterdam, Oxford, 1999), p. 67.

[74] K. S. Hardman, P. B. Wigley, P. J. Everitt, P. Manju, C. C. N. Kuhn, and N. P. Robins, Time-of-flight detection of ultra-cold atoms using resonant frequency modulation imaging, Opt. Lett. 41, 2505 (2016).

[75] J. Stenger, S. Inouye, A. P. Chikkatur, D. M. Stamper-Kurn, D. E. Pritchard, and W. Ketterle, Bragg Spectroscopy of a BoseEinstein Condensate, Phys. Rev. Lett. 82, 4569 (1999).

[76] S. Richard, F. Gerbier, J. H. Thywissen, M. Hugbart, P. Bouyer, and A. Aspect, Momentum Spectroscopy of 1D Phase Fluctuations in Bose-Einstein Condensates, Phys. Rev. Lett. 91, 010405 (2003).

[77] M. Johnsson, S. Haine, J. Hope, N. Robins, C. Figl, M. Jeppesen, J. Dugué, and J. Close, Semiclassical limits to the linewidth of an atom laser, Phys. Rev. A 75, 043618 (2007).

[78] S. S. Szigeti and S. A. Haine, Fundamental limits to cold-atom gravimetry with thermal states (unpublished).

[79] S. Gerlich, L. Hackermuller, K. Hornberger, A. Stibor, H. Ulbricht, M. Gring, F. Goldfarb, T. Savas, M. Muri, M. Mayor, and M. Arndt, A Kapitza-Dirac-Talbot-Lau interferometer for highly polarizable molecules, Nat. Phys. 3, 711 (2007).

[80] R. E. Sapiro, R. Zhang, and G. Raithel, Atom interferometry using Kapitza-Dirac scattering in a magnetic trap, Phys. Rev. A 79, 043630 (2009).

[81] B. Gadway, D. Pertot, R. Reimann, M. G. Cohen, and D. Schneble, Analysis of Kapitza-Dirac diffraction patterns beyond the Raman-Nath regime, Opt. Express 17, 19173 (2009).

[82] W. Li, T. He, and A. Smerzi, Multimode Kapitza-Dirac Interferometry with Trapped Cold Atoms, Phys. Rev. Lett. 113, 023003 (2014).

[83] T. He and P. Niu, Multimode Kapitza-Dirac interferometer on Bose-Einstein condensates with atomic interactions, Phys. Lett. A 381, 1087 (2017).

[84] J. Fekete, S. Chai, S. A. Gardiner, and M. F. Andersen, Resonant transfer of large momenta from finite-duration pulse sequences, Phys. Rev. A 95, 033601 (2017).

[85] V. Guarrera, R. Moore, A. Bunting, T. Vanderbruggen, and Y. B. Ovchinnikov, Distributed quasi-Bragg beam splitter in crossed atomic waveguides, Sci. Rep. 7, 4749 (2017).

[86] N. P. Robins, C. Figl, S. A. Haine, A. K. Morrison, M. Jeppesen, J. J. Hope, and J. D. Close, Achieving Peak Brightness in an Atom Laser, Phys. Rev. Lett. 96, 140403 (2006).

[87] M. T. Johnsson and S. A. Haine, Generating Quadrature Squeezing in an Atom Laser Through Self-Interaction, Phys. Rev. Lett. 99, 010401 (2007). 